\title{
Supporting Special-Purpose Health Care Models via Web Interfaces
}

\author{
James R. Warren, Heath K. Frankel, Joseph T. Noone and Berend-J. van der Zwaag \\ Advanced Computing Research Centre, University of South Australia \\ warren@cs.unisa.edu.au
}

\begin{abstract}
The potential of the Web, via both the Internet and intranets, to facilitate development of clinical information systems has been evident for some time. Most Web-based clinical workstations interfaces, however, provide merely a loose collection of access channels. There are numerous examples of systems for access to either patient data or clinical guidelines, but only isolated cases where clinical decision support is presented integrally with the process of patient care, in particular, in the form of active alerts and reminders based on patient data. Moreover, pressures in the health industry are increasing the need for doctors to practice in accordance with "best practice" guidelines and often to operate under novel healthcare arrangements. We present the Care Plan On-Line (CPOL) system, which provides intranet-based support for the SA HealthPlus Coordinated Care model for chronic disease management. We describe the interface design rationale of CPOL and its implementation framework, which is flexible and broadly applicable to support new health care models over intranets or the Internet.
\end{abstract}

\section{Introduction}

"Information overload" in medicine has long been acknowledged. Medical knowledge is vast and constantly changing, as well as expanding. The doubling time of medical knowledge is currently about 19 years [1], yet a recent survey found that textbooks available to physicians in their workplace are often more than 10 years old [2]. Leaving aside both basic and specialised medical knowledge, a General Practitioner (GP) in Britain is expected to practice in accordance with the contents of numerous policies, referral protocols, government circulars, adverse drug effect warnings, etc. that form a stack 18 inches tall [3]. It is unrealistic to believe that the typical GP has read all these materials; it is a cognitive impossibility that all these rules are accurately operationalized on every patient when each consult lasts just several minutes.

To further exasperate the information crisis for doctors, expectations are rising. One might assume that health care in the $20^{\text {th }}$ century is based on the results of research and consensus among specialists and experts in the various medical fields; however, this is not the case. Only in the past decade has there been a systematic quality control and review of outcomes of treatments [4]. Evidence Based Medicine (EBM) is "the conscientious, explicit and judicious use of current best evidence in making decision about the care of individual patients. The practice of EBM means integrating individual clinical expertise with the best available external clinical evidence from systematic research" [5]. Increasingly, doctors are expected to practice in accordance with clinical practice guidelines that distil EBM findings for "best practice" in quality, uniformity and cost effectiveness.

One approach to overcome the lack of information available to doctors is to replace segments of their reasoning responsibility with expert systems. A problem with this approach lies in the issue of responsibility. Doctors have little use for "Greek Oracle" systems [6]. They must reach their own decisions, for which they, not the machine, are ultimately responsible. Given that the doctor is to remain in the loop, a more subtle problem arises with the human-computer (doctor-computer) interface design. As summarised by Richards [7], lack of attention to HCI issues is identified as a major contributing factor to the poor acceptance of early expert systems [8,9]. Classically, interaction is offered in consultation mode, where the user is asked questions and recommendation is made by the system. Consultation mode has been criticised as inappropriate for user control, visibility and user initiative [10]. There are notable instances of clinical expert systems succeeding in production environments; for example, an antibiotics and other antiinfective management expert system has shown significant cost and health benefits for critically ill patients in a hospital intensive care ward [11]. However, it is significant that this success is observed in an environment where integral clinical information systems have been in place for many years and have become part of the local culture (and that the authors do not call the computer-based tool an "expert system").

The main thrust of medical computing for knowledge delivery in the past few years has been on-line practice guidelines [12], emphasising the provision of decision support information to doctors rather than the use of semi- 
autonomous reasoning agents. Moreover, the use of Webbased architectures for guideline delivery [13] shows obvious benefits in terms of accessibility and platform independence of solutions. Clinical practice guidelines (hereafter clinical guidelines or simply guidelines) are standardised specifications for care developed by a formal process that incorporates the best scientific evidence of effectiveness with opinions of experts in the fields [14]. In general, they have been developed in an effort to reduce health care costs without sacrificing quality and have been shown to improve health care outcomes when followed [15]. To be effective, guidelines need to be integrated into doctors' decision-making process in daily practice [16]. In fact, the highest probability of an effective guideline implementation occurs when patient-specific advice is provided at the time and place of a consultation $[12,15,17]$. Most current guidelines are implemented in printed form, or as direct translations of the printed text-based narratives [13]. Zielstorff views the direct online provision of this printed narrative as the lowest form of on-line guideline. The best outcome is achieved when "the guideline is made accessible through computerbased, patient-specific reminders that are integrated into the clinician's workflow" [12]. To accomplish this, we must integrate the Electronic Patient Record (EPR) and the guidance in a single coherent user interface to the clinical information system.

In this paper, we describe our approach to clinical information system design as illustrated by the Care Plan On-Line (CPOL) system. CPOL supports a specialised model of care, the SA HealthPlus "Coordinated Care" model, using a wide-area intranet based system. CPOL integrates delivery of clinical guidance with the process of performing care coordination for the GP. CPOL relies on use of a client application that can receive any of a wide variety of data/guidance "forms" from the CPOL server. The framework is sufficiently flexible to accommodate the range of chronic diseases managed under SA HealthPlus Coordinated Care and is intended to be sufficiently flexible to support other care models as well with minimal modification.

In the next section, we review trends in GP computing in Australia that provide a context for the present CPOL system, as well as delineating future development directions. We describe the rationale and design of the CPOL user interface. Subsequently, we provide details of the architecture that support CPOL and the directions for development of an even more flexible system. We conclude with a recap of the significance of CPOL and decision support that is integral to the process of care.

\section{Developments in Australian General Practice Computing}

\subsection{6-minute Medicine}

The current government health insurance scheme in Australia rewards GPs for the fast processing of patients. In particular, $79.1 \%$ of Australian GP services billed to Medicare in the June 1995 quarter were declared to take between 6 and 20 minutes [18], corresponding to a particular band in the Medical Benefits Scheme (MBS) whereby the doctors are reimbursed for their time. If one is cynical, she might realize that the economically rational behaviour for the GP that spends most of his time conducting 6-20 minute consults is to manage the consults to be as close to the 6-minute minimum as possible. Hence, we may call this government-encouraged behaviour "6-minute medicine."

Continuing in a cynical vein, the most reliable way to turn around a patient in 6 minutes is to write him a prescription ("script") and send him on his way. It is often said the GPs overprescribe antibiotics [19], and this financial incentive for quick turnaround times could be related. It is impossible to provide counselling in this tight time frame, and so many valuable preventive activities (e.g., smoking cessation therapy, dietary therapy, discussion of family issues) are discriminated against by the financial system. Not surprisingly, the most common clinical software packages on GP's desktops, Medical Director and MIMScript, are tailored to fast, efficient script writing.

\subsection{Coordinated Care}

Although the name may vary among countries (Managed, Community, Shared or Coordinated Care), the fundamental concept is nothing new to the developed world. Care Net Illawarra, an Australian Coordinated Care trial encompassing a region south of Sydney, sets out motivating issues for coordinated care that include [20]:

- GPs referring to higher levels of care because of patient expectations and absence of incentives for GPs to manage

- Poor communications between service providers

- Service providers powerless to solve inter-provider isolation

Perhaps the most fundamental objective is facilitating the proactive planning of interventions instead of a crisis reaction approach.

The SA HealthPlus Coordinated Care trial is a set of ten related projects run under the auspices of a special unit of the SA Department of Human Services as part of a nationwide set of Commonwealth sponsored trials of 
various care management models. The HealthPlus trial focuses on patients with chronic and complex health problems that currently require high service use. Individual projects are based on region and health problem and include diabetes, cardiac, aged care, lung disease, back pain and complex illness. HealthPlus began enrolling patients in July 1997 and runs until the end of 1999, having enrolled and managed some 4000 patients.

\section{Support Process}

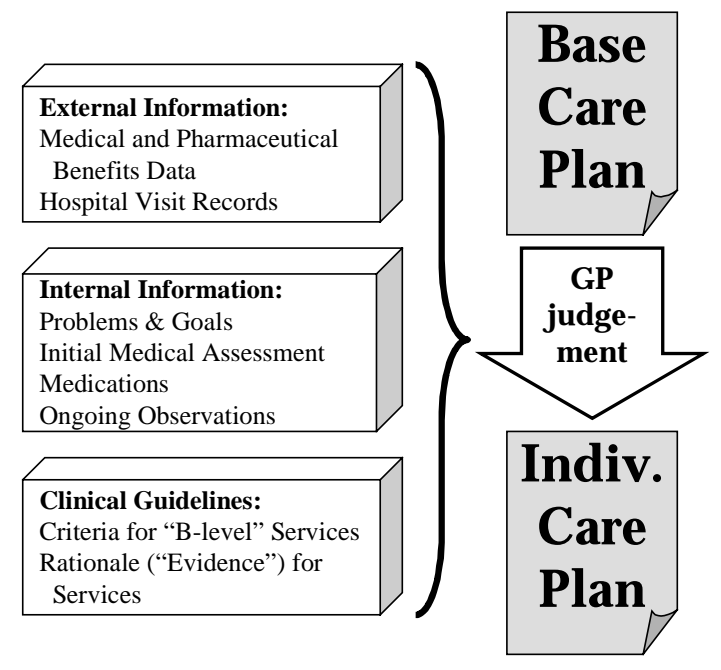

Figure 1. SA HealthPlus Coordinated Care process and its supporting information

Three key elements of the HealthPlus coordinated care model are information, planning and guidance. Figure 1 illustrates the care planning process that is central to HealthPlus. The HealthPlus Unit runs a server that collects external information to support care planning, including area hospital records and Health Insurance Commission (HIC) records of medical and pharmaceutical benefits (and hence services used by the patient). Internal to HealthPlus, data is collected for each patient including a Problem and Goal (P\&G) statement in the patient's own words, an Initial Medical Assessment (IMA), and an upto-date profile of the patient's medications and ongoing clinical observations. The $P \& G$ reminds the doctor of the "whole patient" perspective (more than just the disease state). The IMA is tailored for each project and is developed by that project's Care Mentor Group.

Care Mentor Groups, which include leading area specialists, GPs, nurses and allied health professionals, consolidate best-practice guidance appropriate to each project. The products of the Mentor group include the IMA and criteria for assessing a patient as Mild, Moderate or Severe. A Base Care Plan (BCP) dictating a proactive, 12-month schedule of services is devised for each of the three severity levels. Such services include visits to the GP and specialists, laboratory tests and supporting wellness services (e.g., physiotherapy or dietitian). The Mentors also develop a set of guidelines to advise on criteria for including further optional ("B-level") services in the care plan beyond the BCP. These guidelines include a summary of the rationale and supporting evidence for the health benefits of the services.

Each HealthPlus patient nominates a GP to act as her Care Coordinator. This GP, with the support of a nurse Service Coordinator, is responsible for devising the patients individualised care plan. This care plan starts with the BCP and then is varied according to the GP's interpretation of the patient's needs in accordance with the guidelines from the Mentors. The GP then regularly monitors the patient's progress and periodically (every 3 , 6 or 12 months, depending on the patient's severity level) formally reviews the care plan. The GP is financially compensated for care planning outside of the normal " 6 minute medicine" model, and is expected to invest 45-60 minutes in a care plan creation or formal review session.

Our group has developed the Care Plan On-Line (CPOL) software to provide an intranet-based support tool for the GP's care planning process. CPOL is described in the subsequent sections.

\subsection{Common Health Record Architectures}

Clinical uses of computing are widely perceived as lagging their potential (e.g., in comparison to the use of IT in other sectors) [21]. However, in the past few years it seems the floodgates are finally opening to allow health computing to move from chiefly administrative functions to direct clinical support [22]. As part of this movement, we see the emergence of (often ambitious) government plans for IT in healthcare. For instance, the UK National Health Service has a $£ 1$ billion plan for 1998-2005 period for a comprehensive, personal EPR for every citizen of the UK as well as the availability of up-to-date, on-line health information for patients and the public [23]. In Australia, a Strategic Framework for General Practice Information Management and Technology has recently been put forth [24]. This strategy includes consolidation of the experiences from the Coordinated Care trials as well as introduction of standards for EPR architecture and interchange to facilitate coordination.

A major input to the Australian General Practice EPR architecture is likely to be the Good Electronic Health Record (GEHR) architecture, originally developed under EU funding [25]. The GEHR architecture views each patient record as a collection of transactions, where each transaction conforms to an archetype structure. While GEHR says much about the requirements for information structure to ensure interoperability of systems, it does nothing to prescribe the user interface. Thus, further work 
will be needed to achieve consistent and coherent clinical workstation designs, even if GEHR is adopted. A promising implementation path is employ XML technology such that GEHR transactions map to XML files and archetypes to DTDs. A clinical workstation client, not dissimilar to our CPOL client, could then be devised based on manipulation of these XML files.

\section{Care Planning Interface}

\subsection{The Rationale}

The Care Plan On-Line (CPOL) system is designed to support a General Practitioner (GP) in devising, maintaining and periodically reviewing a 12-month care plan for a chronically ill patient being managed under the SA HealthPlus Coordinated Care model. Several key requirements have particularly shaped the user interface design:

- User control

- Flexibility

- Variable user interest in decision support

- Integration of workflow and decision support

User control is cited as "good tool" principle number one by Cox and Walker [26]. While this demonstrates that it is a "motherhood" principle, it is nonetheless especially relevant for GP users. Doctors in general are, of course, highly educated and highly practiced at making patient care decisions. They are notorious for having large egos. They are very busy. They are classic "experts." It is also known that doctors can be "individualistic" in the sense of showing wild variation (and hence a lack of evidence-based best practice) in the extent to which they favour particular remedies [27]. Our intention with CPOL is to "sell" the Care Mentors practice guidance to the GPs, but we must do it in a framework where the GPs feel they have absolute control. A further support for the need for user control comes from a survey we conducted wherein we had three HealthPlus-affiliated doctors rank the order in which they would review a number of information sources in formulating a care plan. There was no consistent pattern, except that all GPs rated P\&G last behind more traditional clinical data.

Flexibility is, like user control, one of Cox and Walker's good tool principles, however it is also, for many of the same reasons, especially relevant in this context. Flexibility, in a narrow sense, is vital due to variation in doctor opinion. While the medical profession is striving to be evidence based, not all questions have yet been answered satisfactorily and legitimate differences of opinion can exist. For instance, one HealthPlus GP disagrees with the use of Hip-Waist ratio as a risk indicator in cardiac disease despite the recommendation of the cardiac Care Mentor group. If we design the HipWaist ratio as a required field, the GP will not use the system (an excessively strict outcome given the relative important of that statistic).

The CPOL system must also be flexible in a broader sense to deal with the range of diseases managed under the HealthPlus model. There are many examples of effective special-purpose clinical information systems. For instance, in South Australia, Medical Communications Associates (MCA) has developed diabetes and asthma management systems in collaboration with area hospitals, featuring decision support for patient assessment, and patient-specific colour printouts of medication explanations to form part of the patient's care plan [28, 29]. However, these systems were purpose built, from database to user interface, to support the management needs of one particular chronic disease without a framework for adaptation to other diseases. The SA HealthPlus Unit insisted that a single system handle all disease-focused projects. A further issue in HealthPlus is that many patients have more than one major chronic disease, requiring an integration of management approaches. Furthermore, some patients, due to a complex combination of illnesses or extreme age, may warrant a flexible approach to the application of management plans (e.g., while smoking cessation is recommended for "all" chronic lung disease patients, few specialists support its application to lifelong smokers above the age of 85).

GPs will be variable and hard-to-predict in terms of their pattern of use of decision support information. GPs vary in age and experience. None of them will know everything the Mentors would desire about all chronic diseases, but many will know a lot about some chronic diseases as per their individual pattern of self-education. Moreover, while generally very busy, GPs are accustomed to fitting in their learning at odd times. Thus, the decision support features need to stay out of the way of the doctor; however, at any time the GP may recognize a knowledge deficit she wishes to correct and proceed to review a concept (and several related concepts) in depth.

The criterion that most separates the development of CPOL from a simple exercise in good user interface design is the need to integrate the decision support features with the GP's workflow. It is relatively easy to create a repository of stand-alone clinical decision support resources, especially with the Web. The WAX Active library is an example of a standalone hypermedia resource designed to replace a cart of books for satisfying doctors' information needs [3]. The Stanford Health Information Network for Education (SHINE) fulfills a similar purpose, but uses SGML mark-ups of resources to facilitate improved search mechanisms [30]. SHINE is designed to log GPs' information searches and provide confirming exercises suitable for the award of Continuing Medical 
Education (CME) credit, thus providing an innovative motivating factor for the doctor to access the knowledgebase.

In their review paper on information resources in primary care, Westberg and Miller [31] identify the need for "dynamically and judiciously linking questions with answer resources," but fail to recognise the fundamental problem of having decision support systems (DSSs) stand outside of the doctor's workflow. In 1995, Cimino et al. [32] illustrated that Web technology could greatly facilitate integration of legacy systems in a hospital environment and that external decision information resources (Medline) could be provided in the same clinical workstation interface. However, this fails to provide active decision support. The user must still: (a) recognise the knowledge deficit; and (b) take time out to conduct searches to find information to repair the deficit. As cited earlier, Zielstorff [12] finds that the demonstrated successes for clinical guidance systems happen when the system actively alerts the doctor.

Information deficits in HealthPlus fit well with the model of providing active alerts. In many cases the GP, once alerted, may not be surprised that a certain patient merits a HealthPlus service, such as physiotherapy or bone density studies. An important part of the solution is to bring such issues to a busy doctor's attention as they perform the required work of authoring a care plan.

Further elements that shape the design of the CPOL interface include the fact that the patient is the central "object" (fairly obvious, but possible to forget when one gets caught up in the details). The HealthPlus model of care results in an exceptional amount of patient data being collected and made potentially available to the GP. Thus, simply presenting this data in an up-to-date and coherent format is a central goal. Also, for success of the coordinated care trial, key outcomes are (a) a quality care plan in accordance with the Care Mentors' vision as set forth in their guidelines; (b) promulgation of the broad concepts of coordinated care (e.g., getting GPs to think more proactively in chronic disease management); and (c) cost control (the trial aims to be cost-neutral).

\subsection{The Design}

The general interface organisation is around a central patient object (figure 2). Critical elements of the patient record are persistently displayed along the top of the screen, including key disease status indicators that vary from project to project. Subsidiary patient information is organised under tabs - a conventional approach, but the metaphor is not lost on doctors who are accustomed to holding thick folders of patient information. The interaction paradigm emphasises direct manipulation includeing the dragging and dropping of services into the individualised care plan and the ability to manipulate a timeline of service appointments (figure 3). Data viewing and entry is dominated by the ability to enter updates of information in any order (via sliders, spinners and other non-keyboard controls as often as possible) and the lack of required data entry fields. All data display areas, including clinical guidelines, are also data entry areas. A simple vocabulary of icons and colour codes indicates editable and current data, alarming values, clinically indicated services, and links to guidelines.

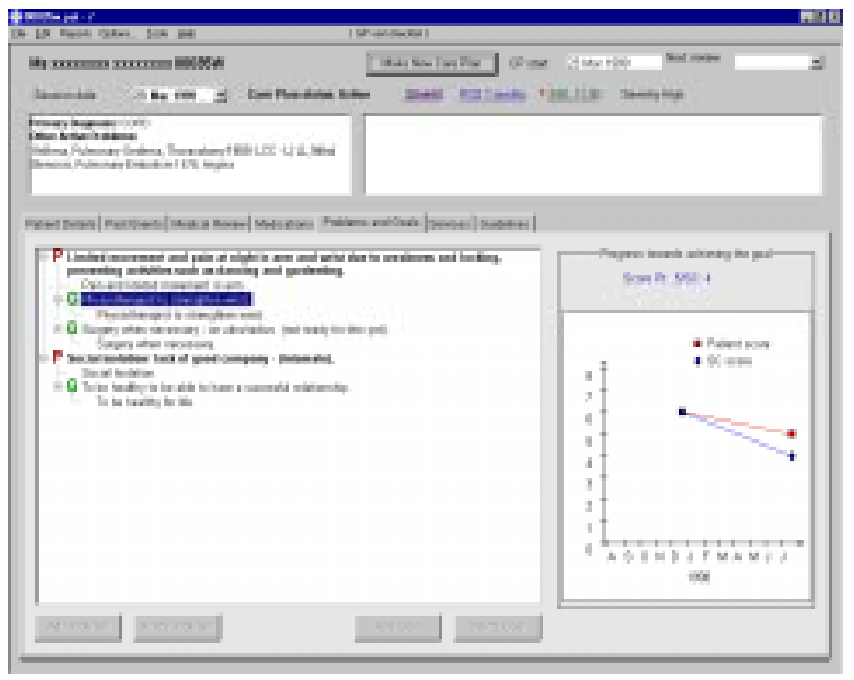

Figure 2. CPOL main screen, showing Problem \& Goal tab with trendline of progress on selected goal.

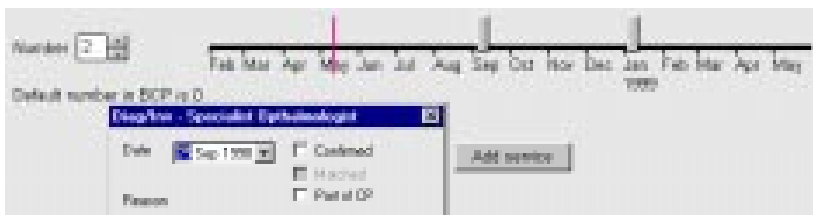

Figure 3. CPOL service timeline control.

Clinical guidelines are available for a selection of key concepts (as defined by the Care Mentor groups) for each major disease managed by HealthPlus. The guidelines are layered (generally into three tabs, although this is reconfigurable) to allow GPs to pursue concepts to the extent of their interest, need and time. At the first level, Status, the GP sees a problem-oriented view of the patient record, with the ability update information directly on the guidelines. On the Status layer, we assume that the GP can understand the issue simply by seeing the data. For instance a trendline showing high and/or rising blood pressure has self-evident significance. System recommendations (e.g., "Check BP every visit") are also displayed on the Status layer. 
If the GP feels uncertain about the significance of the patient data, he can proceed to the Checklist layer (figure 4). Zielstorff [12] considers the highest level of on-line guideline to be one that is adapted to the patient data; however, her vision seems to favour an expert system style of recommendation. We take the approach of preserving the complete guideline rules, as per the printed version, while graphically marking those rules in accordance with the patient status. In this way the GP can follow the Mentors' decision logic, learning it and (if she chooses) accepting it.

If the GP has further interest in the concept after reviewing the Checklist, he may proceed to the Evidence layer. The Evidence layer employs standard HTML browsing technology to access material compiled by the Care Mentors to support the care rationale using both internal and external links.

CPOL provides multiple avenues of access to the clinical guidelines to support their integration with the care planning process. Wherever the clinical observations (such as blood pressure or cholesterol level) are displayed, the system may also include an alert flag indicting "concern" about the related concept (e.g., hypertension for blood pressure). The alert flag may be present at alert (“"” or red dot) or warning/concern (“?”) levels depending on arbitrary logic expressions defined in accordance with the clinical guidelines. Either the observation (whether it is at an alert level or not) or the flag can be clicked to invoke the relevant guideline. Two important sources of alert-prone observations (and related links to guidelines) will be the Initial Medical Assessment (which, by design, surveys key clinical observations) and the critical summary observations persistently visible at the top of the screen. Alert flags are also positioned next to HealthPlus services on the Service tab when they appear to be indicated by the patient data.

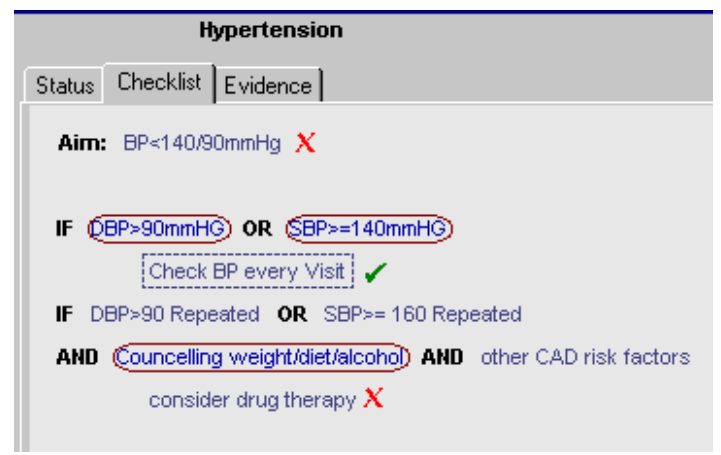

Figure 4. CPOL guideline Checklist layer. Guideline rules are marked in accordance with patient data.

Once in the guideline section, the display includes an index list of all guidelines organised by disease (with the potential to repeat if a guideline pertains to more than one disease) as well as alphabetically. The guidelines also have a hot list of links to closely related guidelines. These features support the GP if he goes into a browsing mode. Moreover, the rightmost tab on the main screen provides direct access to the guidelines so the GP need not look for a flag or related observation if she has a particular information need in mind.

Evaluation of the effectiveness of CPOL is ongoing. An off-line trial of the appropriateness of CPOL triggers in response to respiratory patient data shows that the system gives alerts that are largely in accordance with Mentor decisions and would be of use to GPs in improving their care planning. Field experiments with direct observation of GPs using CPOL are just beginning. Qualitative observations of pilot users show that GPs find the system reasonably intuitive and employ a wide variety of ways to use the system. One unexpected usage path is that GPs often review the guideline index as a checklist of key concepts. A planned experiment will test hypotheses on the pattern of interface usage and user acceptance of the system, as well as its effect on care plan quality.

\section{Care Planning Architecture}

\subsection{Physical Architecture}

To satisfy the Commonwealth's program evaluation criteria, the SA HealthPlus Unit needed to set up a HealthPlus Information System (HIS) to store data regarding the HealthPlus trial. This was done using Progess RDBMS running on an NT machine on a LAN in the Health Commission building within the South Australian Government's Statenet intranet. The HIS was not designed for external access or to satisfy active decision support needs (it does produce summary reports that serve some passive decision support roles). However, the HealthPlus trial includes hypotheses concerning the degree of patient adherence to planned services, as well as costs, that require the HIS to contain the care plan schedule of services for each patient, as well as medical and pharmaceutical data from the Health Insurance Commission and area hospital records. The HealthPlus Unit devised and implements various procedures to update the HIS as necessary, including a Health Level 7 (HL7) data interchange with the state's Oacis data warehouse that spans departments in the major Adelaide metro hospitals.

A CPOL server is implemented as an NT Machine that is both a Web server and a Progress RDBMS client (via ODBC) on the same LAN as the HIS. The CPOL server also, conceptually, has its own local DBMS of auxiliary information for data needed to fulfill decision support 
beyond that in the HIS (for convenience, however, the same Progress database used for the HIS has been extended to provide this auxiliary database function). This architecture is exciting for its manageable and scaleable nature. Separate teams with minimal coordination worry about the "traditional" Management Information System (MIS) issues of the HIS and the Web/DSS issues of the CPOL server, and both are able to leverage massive infrastructural information systems maintained at the state and federal level. Moreover, there is nothing limiting a server like the CPOL server from having a database client relationship to MISs beyond the HIS.

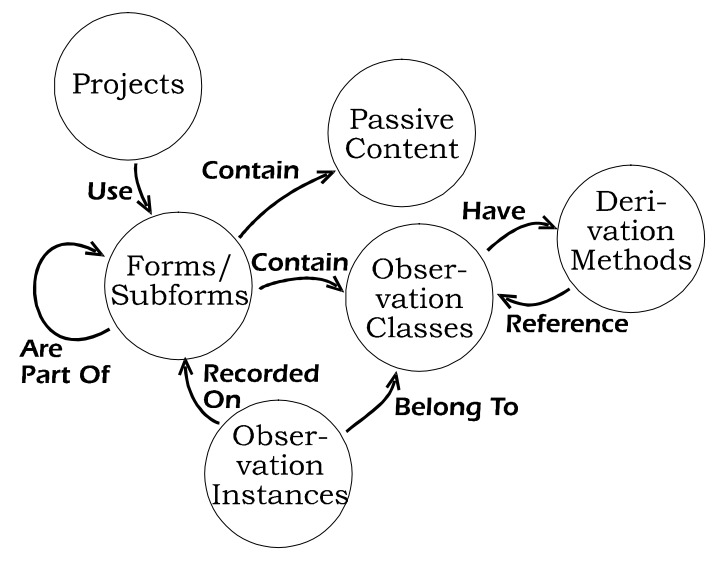

Figure 5. Semantic network of CPOL representation scheme for observations and forms

\subsection{Data Architecture}

The key to integrating decision support and workflow in CPOL is the close relationship between the patient data, "business process" model, and clinical knowledge as illustrated in figure 5 (explained further in [33]). The character of a given disease-specific project is dictated by its choice of forms (unique ones such as the IMA and ongoing assessment, as well as HealthPlus-wide ones such as the Events, Medications and Services forms). When a CPOL client requests a particular patient, the server determines that patient's project membership and then assembles the applicable forms populated with the relevant instances of the clinical observations required for those forms. Nearly all data display/entry information is manifest through these forms, including all the tabs on the main screen and the clinical guidelines. Clinical guideline decision rules and alert flags are also implemented as classes of observation (the same as a blood pressure reading) via the facility to define observations as functions of other observations. All persistent storage is implemented through relational database tables.

The CPOL data structure is sufficiently flexible that administrative forms and clinical guidelines can be up- dated without requiring recompilation of the CPOL server or the clients. An extensive modification, such as the recent addition of hospital records to the Events form, required a recompile of the server (because new SQL commands and processing logic were needed to condition the data from its HIS format to CPOL observations), however, no changes to the CPOL clients were required.

\subsection{CPOL: The Next Generation}

The current CPOL client, developed in Microsoft Visual $\mathrm{C}++$, recognises only a fixed set of user interface objects. Object instances are instantiated on the CPOL server, which interprets the form structures for the requested patient, then are serialised and transmitted to the client over a TCP/IP connection. A further limitation is that the current framework, while flexible, still relies on considerable logic about the model of care being hardcoded in the client.

A new architecture is being developed that will utilise the late binding mechanisms of Java to allow extension of the client with new user interface object classes at any time. Furthermore, this extensibility can be carried deeper to configure dynamically the model of care (the "business process"). Moreover, a decorator design pattern can be used to implement an interface between user interface objects created with standard visual programming tools and the clinical observation framework of the CPOL client.

\section{Conclusions}

We have presented our Care Plan On-Line (CPOL) system as an instance of the use of an Internet/intranet based architecture that projects support for a novel model of medical care to a distributed community of health practitioners. As health expenditures rise across developed countries, so will the pressure on doctors to provide efficient and effective medicine in accordance with the latest findings of medical science. Moreover, there will be an expectation that IT will be used by doctors to provide innovative solutions. We argue that an essential element of such IT solutions is the integration of decision knowledge with the electronic patient record and the process of care. CPOL demonstrates a flexible architecture that provides this integration.

The future of clinical information systems will include increasing use of standard health information architectures to enable interchange of patient data and decision knowledge, and coordination of care. Our research goals are to further demonstrate the effectiveness of CPOL, extend the flexibility of the architecture to accommodate new user interface controls and knowledge and reasoning sources, 
and adapt its architecture to embrace emerging Australian health record architecture standards.

\section{Acknowledgment}

Thanks are owed to the industry collaborators on this work, Medical Communication Associates, The Queen Elizabeth Hospital and the SA HealthPlus Unit of the SA Department of Human Services. This work is supported in part by Australian Research Council Collaborative Grant C19700395 and Australian Postgraduate Award (Industry) AP96073.

\section{References}

[1] Smith, R. (1996). What clinical information do doctors need? BMJ 313(7064), 1062-1068.

[2] Hubbs, R. et al. (1997). The Stanford health information network for education: integrated information for decision making and learning, Proc. AMIA Annual Fall Symp., 505508.

[3] Hanka, R., O’Brien, C., Heathfield, H. \& Buchan, I. (1999). WAX ActiveLibrary; a tool to manage information overload, Proc. 32 ${ }^{\text {nd }}$ Hawaii Int'l Conf. System Sciences, IEEE Computer Society Press.

[4] Rosenberg, W. \& Donald, A. (1995) Evidence based medicine: an approach to clinical problem-solving. $B M J$ 310, 1122-1126.

[5] Sackett, D., Rosenberg, W., Gray, J., Haynes, R. \& Richardson, W. (1996). Evidence based medicine: what it is and what it isn't. BMJ 312, 71-72.

[6] van Bemmel, J. \& Musen, M. (1997). Handbook of Medical Informatics, Heidelberg: Springer.

[7] Richards, D. (2000). User-centred and driven knowledgebased systems for clinical support using ripple down rules. Proc. $33^{\text {rd }}$ Hawaii Int'l Conf. System Sciences, IEEE Computer Society Press (to appear).

[8] Langlotz, C. \& Shortliffe, E. (1983). Adapting a consultation system to critique user plans. Int'l J. ManMachine Studies 19, 479-496.

[9] Salle, J. \& Hunter, J. (1990). Computer-user cooperation issues for knowledge-based systems: a review, Tech. Rpt. AUCS/TR9003, Aberdeen University.

[10] Baroff, J., Simon, R., Gilman, F. \& Shneiderman, B. (1988). Direct manipulation user interface for expert system. In, Hendler, J., (ed.) Expert Systems: The User Interface, Albex Publishing.

[11] Evans, R., Pestonik, S. et al. (1998). A computer-assisted management program for antibiotics and other antiinfective agents. NEMJ 338, 232-238.

[12] Zielstorff, R. (1998). Online practice guidelines, JAMIA 5, 227-236.

[13] Liem, E., Obeid, J., Shareck, E., Sato, L. \& Greenes, R. (1995). Representation of clinical practice guidelines through an interactive World-Wide-Web interface, JAMIA, Symposium suppl., 223-227.

[14] Leape, L. (1990). Practice guidelines and standards: an overview, Quarterly Review Bulletin '90 16, 42-49.
[15] Grimshaw, J. \& Russel, I. (1993). Effect of clinical guidelines on medical practice: a systematic review of rigorous evaluations, Lancet 324, 1317-1322.

[16] Lam, S. (1994). Implementation and evaluation of practice guidelines, JAMIA, Symposium suppl., 253-263.

[17] Shiffman, R. (1997). Representation of clinical practice guidelines in conventional and augmented decision tables, JAMIA 4, 382-393.

[18] Commonwealth Department of Health and Family Services (1997). General Practice in Australia. Canberra, March.

[19] Lode, H. (1991). Respiratory tract infections: when is antibiotic therapy indicated? Clin. Therap. 13(1), 149-156.

[20] Foulston, J. (1997). Care Net Illawarra: A Coordinated Care Trial. Dapto, NSW, Australia: Care Net Illawarra.

[21] Kondro, W. (1999). Canada must update patient records. The Lancet 353, 568.

[22] Anderson, J. (1997). Clearing the way for physicians' use of clinical information systems. CACM 40(8), 83-90.

[23] NHS Information Authority (1999). An information strategy for the modern NHS. URL: www.nhsia.nhs.uk

[24] General Practice Computing Group (1999). Strategic Framework: General Practice Information Management and Technology. Canberra: Commonwealth Dept. Health and Aged Care.

[25] The GEHR Foundation (1999). The Good Electronic Health Record: an innovation in software engineering for the benefit of patients. URL: www.gehr.org

[26] Cox, K. \& Walker, D. (1993). User Interface Design, 2nd ed., Prentice Hall.

[27] Wall, E. (1993). Practice Guidelines: promise or panacea? J. Family Practice, 37(1), 17-29.

[28] Adams, R., Ruffin, R., Smith B., Campbell, D. \& Dippy, S. (1997). Problems and some solutions in adapting clinical practice guidelines for Asthma patient management into a computerised management system. The Western Region asthma project (Wrapp). Proc. Asia Pacific Assoc. of Med. Informatics / Health Informatics Soc. of Australia (APAMI-HIC97) Joint Conf. Brunswick East, Victoria: Health Informatics Society of Australia.

[29] Bruand, W., Dippy, S., Schloeffel, P., Sickles, D. \& Dalidowicz, L. (1997). A specialised electronic network to coordinate expert care of diabetes. Proc. Asia Pacific Assoc. of Med. Informatics / Health Informatics Soc. of Australia (APAMI-HIC97) Joint Conf. Brunswick East, Victoria: Health Informatics Society of Australia.

[30] Godin, P., Hubbs, R., et al. (1999). A new instrument for medical decision support and education: the Stanford health information network for education. In, Proc. $32^{\text {nd }}$ Hawai'i Intn'l Conf. Systems Sciences (HICSS-32), IEEE Computer Society Press.

[31] Westberg, E. \& Miller, R. (1999). The basis for using the Internet to support the information needs of primary care. JAMIA 6, 6-25.

[32] Cimino, J., Socratous, S. \& Clayton, P. (1995). Internet as clinical information system: application development using the world wide web, JAMIA 2, 273-284.

[33] Warren, J., Beliakov, G., Noone, J. \& Frankel, H. (1999). Chronic disease coordinated care planning: flexible, taskcentered decision support. Topics in Health Information Management (to appear, November). 